\section{$\underset{\substack{\text { hommes } \\ \text { \& migrations }}}{ }$}

\section{Hommes \& migrations}

Revue française de référence sur les dynamiques

migratoires

\section{$1278 \mid 2009$}

Histoire des immigrations. Panorama régional

\title{
Khaled : Liberté
}

\section{François Bensignor}

\section{OpenEdition \\ Journals}

Édition électronique

URL : http://journals.openedition.org/hommesmigrations/268

DOI : 10.4000/hommesmigrations.268

ISSN : 2262-3353

\section{Éditeur}

Musée national de l'histoire de l'immigration

\section{Édition imprimée}

Date de publication : 1 mars 2009

Pagination : 250-255

ISSN : 1142-852X

Référence électronique

François Bensignor, « Khaled : Liberté », Hommes \& migrations [En ligne], 1278| 2009, mis en ligne le 29 mai 2013, consulté le 21 décembre 2020. URL : http://journals.openedition.org/hommesmigrations/ 268 ; DOI : https://doi.org/10.4000/hommesmigrations.268

Ce document a été généré automatiquement le 21 décembre 2020.

Tous droits réservés 


\title{
Khaled : Liberté
}

\author{
François Bensignor
}

1 Avec la publication de son nouveau disque, le personnage de vedette internationale à la vie dissolue et aux frasques imprévisibles que les médias lui ont taillé sur mesure s'éloigne de plus en plus de Khaled. À présent installé au Luxembourg, le chanteur, qui est entré dans sa cinquantième année, semble avoir tiré un trait sur une ambiguïté qu'il avait cultivée, plus par jeu que par conviction, autour du personnage sulfureux hérité de ses jeunes années raï. Il est vrai que la presse people, qui n'aime rien tant que salir les noms de celles et ceux dont le statut d'idole dure un peu trop longtemps, lui a fait payer au prix fort une désinvolture trop longtemps affichée derrière son éternel sourire.

"Khaled passe pour un alcoolique alors qu'il est sobre depuis une douzaine d'années. On lui a fait une réputation lamentable qu'il ne mérite pas, même s'il a fait quelques bêtises dans le passé. Il traîne cela comme un boulet et ce n'est pas juste", estime Martin Meissonnier, homme de média et producteur artistique de son nouvel album, le premier à avoir fait venir jouer en France le Cheb, alors roi du raï, en 1986. "Les médias se sont acharnés sur lui, parce qu'il ne les maîtrisait pas assez dans leurs conventions françaises, poursuit-il. Dans l'émission de Fogiel, il s'est retrouvé piégé... En disant 'Qui aime bien châtie bien' [à propos de sa femme qu'il avait frappée et qui avait porté plainte], il croyait faire une blague. Il ne s'est pas rendu compte que c'était la dernière chose à dire dans ce contexte... Mais dans la réalité, sa femme vit toujours avec lui : elle est son manager... Et quand on connait bien l'histoire, on se rend compte que l'on est face à des médias totalement indécents."

\section{La force de la scène capturée en studio}

3 À plus d'un titre, on peut considérer le nouvel album de Khaled comme la volonté salutaire de réhabiliter une très grande voix de la musique populaire en revenant délibérément à la source authentique de son art. "Raïkoum", "La liberté", "Yamina", ces trois énormes succès du début des années 1980 n'avaient, jusqu'à leur réenregistrement pour ce disque, d'autres témoignages en studio que les versions originales faites à Oran sur ordinateur, avec les pauvres moyens d'il y a trente ans, la voix calée entre synthétiseur et boîte à rythmes. Leurs versions réinterprétées par le 
groupe de Khaled au complet donnent à ces morceaux de bravoure une dimension orchestrale qui les sublime. Sans rien trahir de l'énergie authentique du raï, Martin Meissonnier réalise pour cet album un fabuleux travail de restauration artistique, encouragé par la maison de disques AZ/Universal.

"Je me suis aperçu que, dans le raï, à part quelques vieilles cassettes de Khaled, je ne connais pas un disque qui n'ait été enregistré sur un ordinateur. Les concerts sont toujours meilleurs que les enregistrements et surtout dans le cas de Khaled, qui tire l'orchestre avec sa voix de façon incroyable. Alors, évidemment, quand il place son chant en studio sur la base instrumentale, c'est toujours moins bien." Il a donc l'idée d'enregistrer Khaled avec les huit musiciens du groupe qui l'accompagnent généralement sur scène: Maurice Zemmour à la basse, Julien Tekeyan à la batterie, Amar Chaoui aux percussions, Mohamed Berkane Krachai au violon, Abdelhoued Zaim au oud, Alain Perez à la guitare, Moustapha Didouh et Elie Chemali aux claviers.

5 Certains d'entre eux, qui jouent avec Khaled depuis des dizaines d'années, n'avaient jusqu'alors jamais eu l'occasion de faire un album complet avec lui. Chargés du casting des musiciens en studio, les producteurs artistiques anglais et américains (Steve Hillage, Don Was) de ses albums grand public recrutaient parmi les pointures qu'ils avaient l'habitude de fréquenter. "Il y a eu quelques bons titres, mais c'est sur scène que Khaled m'a toujours intéressé, explique Meissonnier. Je trouvais dommage qu'on l'ait poussé à faire des choses qui ne lui ressemblent pas." C'est donc une stratégie d'album live qu'il met en œuvre afin de capturer cette étincelle qui rend les prestations publiques de Khaled si captivantes.

\section{Retour aux racines oranaises et marocaines}

Une fois déterminé l'esprit de l'interprétation, il s'agit de dégager une cohérence à travers un choix de chansons. Khaled, qui n'avait plus enregistré d'album depuis $Y a-$ Rayi, paru en 2004, avait eu largement le temps d'accumuler les idées. "Il y avait beaucoup de maquettes, dans plein de genres différents, jusqu'à des morceaux raggatone, se souvient Meissonnier. Il fallait faire un choix. On a opté pour un album qui retourne aux racines oranaises et marocaines."

7 Nous voici donc projetés aux alentours de l'année 1985. Le raï, dont l'explosion est encore circonscrite à l'Algérie, est le grand phénomène culturel marquant la jeunesse. Oran sert de laboratoire et de rampe de lancement à la créativité des nouveaux artistes, en rupture avec la chanson traditionnelle qu'écoutent leurs parents. Alors que seule celle-ci a droit de cité sur les ondes des radios, dans la nuit oranaise des cabarets de la corniche et dans l'intimité des fêtes privées, garçons et filles (cheb et chaba) du raï laissent libre cours à leur imagination, chassant la frustration. À 25 ans, Cheb Khaled affirme qu'il chante "le sexe, l'alcool, les femmes, les thèmes que nos générations n'osaient pas aborder, par respect, devant nos parents. Tout ce que l'islam prohibe et qui soulève la colère des intégristes".

8 Sa cassette Hada Raikoum est vendue à 3 millions d'exemplaires.

9 Jeune producteur artistique qui vient de faire découvrir au public européen deux artistes majeurs du Nigeria, Fela et King Sunny Adé, Martin Meissonnier s'entiche de cette musique nouvelle. Il vient de découvrir Khaled sur une cassette qu'un copain algérien lui a fait écouter, une réalisation des frères Rachid et Fethi Baba Ahmed, 
producteurs dont la touche artistique donne un cachet incomparable aux musiques enregistrées dans leur studio de Tlemcen. "J'ai trouvé ça génial et j'ai décidé d'aller les voir. Au studio de Tlemcen, j'ai rencontré Cheb Sahraoui, qui m'a servi de guide à Oran."

Il revoit encore la scène de sa première rencontre avec le jeune roi du raï: "Des tentes étaient dressées sur les toits en terrasse et j'ai assisté à une joute vocale démentielle entre Khaled et Sahraoui, qui étaient alors parmi les plus belles voix d'Oran. À l'époque, Khaled chantait dans les mariages toute la nuit.

Il commençait pour les hommes et, à partir de 3 heures du matin, changeait de répertoire et chantait pour les femmes. Il était assis en tailleur et s'accompagnait sur deux synthétiseurs : un Kawai 900 qui produisait un son genre cornemuse et un Crumar pour faire des nappes; avec lui, un violon, une basse, une derbouka, un târ et une guitare."

\section{Transit par Bobigny vers l'international}

Le voyage à Oran de Martin Meissonnier allait aboutir à l'organisation du fameux festival raï de Bobigny, le 23 janvier 1986, premier événement qui donna à cette musique une visibilité internationale.

"Ce concert en banlieue n'avait bénéficié que d'une maigre campagne de publicité, mais son succès a été totalement inattendu, se souvient-il. Les 2000 places de la MC93 ont été prises d'assaut et les 2000 personnes restées dehors essayaient de se frayer un chemin à l'intérieur. Khaled n'avait pas de passeport, mais sa légende l'avait précédé... J'avais très envie de travailler avec lui, mais il était tenu par deux producteurs, K. Feddal (Horizon-Music) à Barbès et Missoum Zed El Youm (MCPE) à Oran. Tous deux revendiquaient un contrat d'exclusivité et se pirataient mutuellement. C'était une situation inextricable et j'ai préféré laisser tomber..."

14 Pendant deux ans, rien ne se passe. "Et puis un jour, je reçois un coup de fil de Safy Boutella qui cherchait un producteur pour l'aider à réaliser un disque de Khaled en studio. Il était tombé sur la bonne personne..." Ce disque était financé par le gouvernement algérien. Le lieutenant-colonel Hocine Snoussi, qui dirigeait l'office Riadh el Feth, organisme fondé sous la présidence de Chadli pour la consommation de luxe et une certaine culture permissive, était en charge de la production de cet album. Il l'avait déléguée en France à Charles Talar et Didier Barbelivien. "C'était incroyablement agréable de travailler avec eux, se souvient Martin. Ils nous ont fait entièrement confiance, sont venus au début et à la fin de l'enregistrement. Et ils étaient contents : 'Merci, au revoir !'”

15 Kutché, puisqu'il s'agit bien du chef-d'œuvre publié en 1988, allait révéler Cheb Khaled au public international.

\section{Les chansons de Liberté : revue de détail}

\section{“Hiya Ansadou” (Allez, on s'en va)}

16 Cette composition de Khaled remonte à l'année 1983. Avec ses musiciens, il jouait dans un cabaret très tard dans la nuit et le public n'arrêtait pas d'en redemander. Épuisé, Khaled entonne alors une mélodie sur ces simples paroles : "On s'en va! On est parti!" Les musiciens le suivent et le résultat est ce prodigieux morceau de raï. "Quand on a commencé à travailler sur l'album, Khaled est venu dans mon studio avec son violoniste, son joueur de oud et un joueur de derbouka, explique Martin Meissonnier. Je leur ai dit : 'Jouez 
tous les bons morceaux qui vous passent par la tête. Laissez-vous aller!' Et à un moment ils se sont retrouvés sur ce thème. J'ai construit la chanson à partir de ce qu'ils ont joué lors de cette première session en y ajoutant des cordes égyptiennes."

\section{"Raïkoum" (Votre loi, votre opinion)}

17 Cet hymne, emprunté au répertoire de feu la grande Cheikha Rimitti, bénéficie d'un prodigieux lifting orchestral. Posée sur son accordéon, l'ample voix de Khaled module une introduction parsemée de touches de violon. Puis la chaleur des claquements de mains lance un groove infiniment raï qu'entraîne la mélodie bondissante du oud. La vitesse de croisière atteinte, la voix de Rita Marley sème un gazon reggae sur le refrain. "Khaled avait enregistré une version de cette chanson en Jamaïque, explique Martin. On a récupéré les chœurs sur l'enregistrement de cette session."

\section{"Ya Bouya Kirani” (Père, dans quel état je suis)}

18 Cette chanson d'amour, dans le plus pur style de la poésie populaire arabe, est l'œuvre de Blaoui Houari (né en 1926), l'un des premiers grands modernisateurs de la musique bédouine oranaise.

\section{"Gnaoui"}

19 Signé Khaled, ce morceau rappelle ses tous premiers pas dans la musique professionnelle. Vers l'âge de 12 ans, il avait fondé le groupe Noujoum El Khams (Les Cinq Étoiles) sur le modèle de Nass El Ghiwan, inventeurs du folk berbère marocain, dans lequel ils introduisirent des éléments de la musique jouée par les Gnaoua. Khaled donna sa première prestation au théâtre régional d'Oran en 1973 avec son groupe, qui disparut deux ans plus tard.

\section{“Zabana"}

20 Autre chanson signée Blaoui Houari, ce raï plein d'émotion commence lentement, avant de s'échapper dans une ambiance jazzy sur les belles notes du piano d'Elie Chemali. Le texte évoque le premier condamné à mort oranais du temps de la colonisation française. Parti rejoindre le maquis, Ahmed Zabana fut capturé le 8 novembre 1954, lors d'une des premières batailles pour la libération de l'Algérie.

21 Dans sa cellule de la prison de Barberousse où il a été transféré à Alger, l'homme voit défiler sa vie. Il laissera une lettre émouvante à ses parents avant d'être guillotiné en 1956.

\section{"La liberté"}

Ce grand tube de Khaled sonne comme jamais, avec ses trois fabuleuses minutes d'introduction accordéon/voix et le synthétiseur analogique purement raï. "Quand on a commencé l'enregistrement, Khaled voulait absolument que je lui trouve un Kawai 900 pour reproduire le son d'époque. Je n'ai pas réussi à retrouver le même modèle, mais 
c'est un équivalent", raconte Martin Meissonnier. Le texte à double sens de ce grand tube évoque la liberté d'un homme qu'une femme garde attaché à elle par un sortilège.

"Deux ans de souffrance et après en France", s'exclame le chanteur. Or deux ans, c'est la durée du service militaire en Algérie...

\section{"Soghri" (Mon enfance, ma jeunesse)}

Cette jolie chanson sur l'enfance, cosignée avec Koubali, se développe en douceur, caressée au reflux du refrain par le souffle des cordes, avant de s'élever toujours plus haut.

\section{“Sidi Rabbi" (Mon Dieu)}

Ce morceau de pur raï, dont on doit la musique à Hatif Mohamed, est construit dans le meilleur style à l'ancienne. Invitation à la danse dans un esprit soufi, c'est une chanson de repentance où le chanteur demande pardon à Dieu pour toutes ses bêtises.

\section{"Yamina"}

Rêve de femme idéale signé Wahby Ahmed, cette chanson démarre sur la rondeur veloutée de violons égyptiens, dont l'opulente section habille neuf titres de l'album, puis se transforme en un raï pêchu à la pureté poignante. La voix de Khaled détache les mots à la manière d'un rap au flow typiquement oranais. Des cuivres viennent ponctuer la montée en puissance de la chanson, laissant s'envoler une trompette latino. "La voix, le violon, le oud, la basse et la batterie ont été enregistrés en une seule prise. C'est une vraie performance de boxeur : j'adore !" commente Meissonnier.

\section{“Papa”}

C'est la seule chanson en français sur cet album, la seule aussi qui tire plutôt du côté de la variété. Évoquant la mémoire de son père, Khaled y exprime toute la puissance émotionnelle de sa voix.

\section{"Sbabi Ntya" (Tu es la cause)}

Une belle chanson d'amour tourmenté coécrite avec Zaim Abdelwaheb. Sa rudesse raï est adoucie par la caresse des violons égyptiens.

\section{"Ya Mimoun"}

Pour la première fois, Khaled s'essaye sur un air typiquement gnaoua. Il chante la longue intro voix et gumbri avec la conviction d'un maalem. Une guitare pop fait démarrer l'orchestre: "Un clin d'œil à Led Zeppelin", glisse Martin Meissonnier malicieusement. L'ardeur s'installe en une superbe ambiance de montée progressive, jusqu'à la transe finale. 


\section{RÉSUMÉS}

En préservant l'énergie de la scène pour l'enregistrement du nouvel album de Khaled, Liberté, le producteur artistique Martin Meissonnier redonne à certains de ses plus grands succès une profondeur jamais encore captée sur disque.

Ce beau travail ainsi que le choix du répertoire invitent à revisiter certains moments clés de la carrière du chanteur. 